\title{
Magneto electret State of Porous Polypropylene and Investigation on Surface Charge Characteristics
}

\author{
Pragya Ojha ${ }^{1}$, M. S. Qureshi ${ }^{2}$, M. M. Malik ${ }^{3}$ \\ ${ }^{1,2,3}$ (Electret research laboratory, Department of Physics, MANIT, Bhopal 462051, India)
}

\begin{abstract}
This paper reports preparation of porous polypropylene magneto electret and its investigation on its surface charge characteristics. The electrets were prepared under the combined action of heat and magnetic field. Influences of polarizing temperature and magnetic field on MEs state of polypropylene were studied. Five different forming temperatures and five different magnetic field strength were used for the surface charge study of magnetoelectrets. Surface charge was found to be of the order of $10^{-10} \mathrm{C}$. Negative iso charge was found in all the magnetoelectrets. The decay of surface charge was studied continuously for 30 days and corresponding time constant were calculated.
\end{abstract}

Keywords - Dielectric, Magnetoelectrets (MEs), porous polypropylene, magnetic field, surface charge.

\section{INTRODUCTION}

Dielectric samples showing persistent polarization is termed as 'Electrets' [1] in analogy with magnet because in many ways an electret is the electrostatic counterpart of magnet. The first electret resembling Heaviside's was prepared by Eguchi [2] by solidifying the molten mixture of carnauba wax and resin in an intense electric field. In an electret, the dielectric is in state of persistent polarization, i.e. the polarized state continues to stay in the dielectric for considerably long time even after the removal of polarizing field. Electrets are classified depending on the physical agency that produces polarization in dielectric under the influence of an external field. The various types of electrets known so far are thermo, photo, radio, cathode, electro and magneto etc. In 1964, Bhatnagar [3] while observing the effect of magnetic field on carnauba wax for detecting Hall Effect, found that magnetic field in conjunction with heat energy (physical agency), could bring about the polarization in carnauba wax.

The study of initial charge, i.e surface charge measured immediately after the electret formation, its subsequent reversal in polarity and decay with time and the amount of residual charge (final charge) almost permanently retained by it, has helped a great deal in understanding the electret state in the dielectric. The method of study of charge trapped within the electreted dielectric, by the modern technique of thermally stimulated current (TSC), [4] has added much more to our knowledge about the electret state. Thus the study of surface charge is one of the basic and important studies; hence it is undertaken for the porous polymers polypropylene for the present work. From the study of surface charge on magnetoelectret, Khare and Bhatnagar propounded a "Two charge Theory" [5] analogous to the two charge theory of Gross and Gemant [6] on thermoelectret. According to the two charge theory on magnetoelectret, the net surface charge is the resultant of two types of charges known as the 'isocharge' and the 'idiocharge'. The isocharge is the charge of the same sign on the surface of electret which may be +ve or -ve depending upon the substance while idiocharge is a charge due to polarization of the dielectric which has opposite charges on the opposite surfaces of electret. The two charge theory which explains the magnetoelectret surface charge is later found to be capable of explaining several phenomena associated with the magnetoelectret state in the dielectric, such as change in dielectric constant, refractive index susceptibility etc. In present study, The surface charge study on porous polypropylene magneto electrets consists in the study of initial charge on either surface of the sample and its dependence on various forming parameters, such as strength of the magnetic field, the constant elevated temperature subsequent measurement of surface charge is done continuously for about a month, to study the possible reversible of charge, its decay with time and the charge finally retained by the sample. These studies give an assessment of strength, stability and retentivity of charge, besides providing us with information regarding the polarization process responsible for the induction of magnetoelectret state in the substance. The results of surface charge study are discussed in the following sections.

\section{Material And Method Of Preparation}

Amorphous or crystalline polymers when stretched, show voids and behave like porous materials. These interstitials sites provide ideal locations for trapping of electric charges. It is established that such materials have increased electrical and mechanical strengths. To demonstrate the above fact polypropylene sheet of $3 \mathrm{~mm}$ with $50 \%$ porosity is used for the preparation of magnetoelectrets and is cut in the form of pellets 
having radius $0.75 \mathrm{~cm}$ and $2.4 \mathrm{~mm}$ thick dimensions. Magnetoelectrets were prepared by following the method already described by Khare and Bhatnagar [3]. Electromagnets of Polytronic Corporation (Mumbai, India), Type-HEM150, SRNo.: 9 and 35 were used to provide the magnetic field. Sample was kept inside the custom made sample holder with heating arrangement. The holder containing the disc shaped sample was kept in the magnetic field in such a way that the circular faces became perpendicular to the magnetic field. The procedure involves heating the sample to a constant desired temperature and the application of the magnetic field at that temperature for a constant duration of time. After that sample was cooled in the presence of magnetic field till they come to room temperature. Series of magnetoelectrets have been prepared by varying the forming magnetic field, from $0.75 \mathrm{~T}$ to $1.5 \mathrm{~T}$. The forming temperature is varied from $70^{\circ} \mathrm{C}$ to $110^{\circ} \mathrm{C}$.

\section{Surface Charge Measurement}

The charge developed on the magnetoelectrets surfaces were measured with the help of lifted electrode method [6] Lifted electrode is based on electromagnetic induction. The measuring device consists of a dissectible type of condenser, which has one fixed heavy plate and the other plate is movable. The lower plate fixed plate acts as a platform for the electret and is earthed. The surface of the electret is first brought about in contact with the surface of the movable plate of the condenser, while doing so it is earthed. It is than de earthed and allowed to move away a fixed distance in the field of the electret. The induced charge on the movable electrode is measured by Kithely $610 \mathrm{C}$ electrometer.

\section{RESULTS AND DISCUSSION}

\subsection{Surface Charge Characteristics}

Table 1 depicts the initial surface charge density of different magnetoelectrets at room temperature. It can be observed that negative surface charge was developed on both the surfaces of magnetoelectrets of porous $\mathrm{PP}$, irrespective of strength of field applied and forming temperature. The final charge attained after initial decay over a short period, are observed to be of the order of $10^{-10} \mathrm{Coul} / \mathrm{cm}^{2}$ in all samples. The formation of surface charge on the magnetoelectrets of porous PP can be described as follows. As mentioned earlier that heat and magnetic field are two main factors responsible for the magnetoelectret state in dielectrics. The heating of dielectric causes the appearance of free charges in them. Thermal dissociation and recombination would lead to an equilibrium condition in which at certain temperature there would always be some charge pairs. The role of magnetic field in the formation of magnetoelectret state in dielectric is not known completely but its effect on the nature and magnitude of initial surface charge and their persistency can very well be seen. The magnetic field may cause the charge separation. These separated charges, after the removal of magnetic field would soon get neutralized, unless it is visualized that the charges are trapped. It seems that the charges, while drifting towards the surfaces under the influence of heat and magnetic field, get trapped. The existence of trap sites in dielectric might be due to impurities or even magnetic field itself, which may be responsible to create them. Such an assumption is not unlike as Haswgawa [7] has shown that the presence of magnetic field gives rise to an additional impurity band in semiconductors, which may not be there prior to field application. The presence of trap sites may also be visualized at the inter faces of the two phases monomer and polymers or polymers and plasticize. These trap sites helps to prolong the persistence of charge.

Polypropylene used for the preparation of magnetoelectret is semi crystalline and porous in nature, having many structural defects conducive to create a large number of deep traps, which are suitable for production of stable electret. This is due to the characteristic ability of deep traps, to store the charge for longer period. From the initial surface charge density data, the contribution of iso-charge and idio-charge components to surface charge density was calculated by the method given by Khare and Bhatnagar [5]. The calculated data is given in Table 2. It can be seen that the magnitude of idio-charge density is very small as compared to that of iso-charge. Therefore we can say that the observed surface charge may possibly be a negative iso-charge. The iso charge may be due to an excess charge trapped near the surfaces of the dielectric. It is similar to fictional charge where mechanical work (rubbing) between the surfaces in contact gives rise to charge exchange. In magnetoelectret it is the heat energy instead of mechanical work which seems to cause charge transfer between the dielectric and the metal (aluminum foil) in contact with the dielectric during preparation. 
Table 1

Variation in Surface Charge Density with Forming Parameters

\begin{tabular}{c|c|c|c|c|c|c}
\hline \multirow{2}{*}{$\begin{array}{c}\text { Forming } \\
\text { Magnetic } \\
\text { fields in Tesla }\end{array}$} & \multicolumn{5}{c}{ Temperature } \\
\hline \multirow{4}{*}{} & \multicolumn{2}{|c|}{$70^{\circ} \mathrm{C}$} & \multicolumn{2}{c}{$80^{0} \mathrm{C}$} & \multicolumn{2}{c}{$100^{\circ} \mathrm{C}$} \\
\cline { 2 - 7 } & $\sigma_{\mathrm{N}} \times 10^{-}$ & $\sigma_{\mathrm{S}} \times 10^{-}$ & $\sigma_{\mathrm{N}} \times 10^{-}$ & $\sigma_{\mathrm{S}} \times 10^{-}$ & $\sigma_{\mathrm{N}} \times 10^{-}$ & $\sigma_{\mathrm{S}} \times 10^{-}$ \\
0.75 & ${ }^{10} \mathrm{C} / \mathrm{cm}^{2}$ & ${ }^{10} \mathrm{C} / \mathrm{cm}^{2}$ & ${ }^{10} \mathrm{C} / \mathrm{cm}^{2}$ & ${ }^{10} \mathrm{C} / \mathrm{cm}^{2}$ & ${ }^{10}{\mathrm{C} / \mathrm{cm}^{2}}^{10}{ }^{10} / \mathrm{cm}^{2}$ \\
\cline { 2 - 7 } 0.85 & -0.324 & -0.844 & -0.324 & -0.844 & -0.389 & -0.714 \\
0.95 & -0.519 & -0.649 & -0.779 & -0.389 & -0.259 & -0.389 \\
1.3 & -0.519 & -0.584 & -1.038 & -0.584 & -1.623 & -1.493 \\
1.5 & -0.779 & -1.428 & -1.233 & -1.818 & -0.389 & -0.584 \\
\hline & -0.909 & -0.584 & -0.519 & -0.779 & -0.389 & -0.714 \\
\hline
\end{tabular}

\subsection{Effect of Magnetic Field and Temperature on Surface Charge Density}

Table3 \& Table 4 showing variation of surface charge density of north and south surfaces with magnetic field and temperature can be explained by the conduction process in the polymer. It has been established that the conduction process in plastic is ionic rather than electronic. These ions arise from impurity included in the structure, where the impurity includes constitutes of different molecular weights because of the combined action of heat and magnetic field, microscopic displacement of ions takes place. At the time of cooling, in the presence of magnetic field these ions get trapped and frozen inside or on the surface of crystallites, giving rise to space charge polarization. This type polarization contributes to the establishment of isocharge in nonpolar polymers (such as polypropylene) Dipolar polarization is not expected in polypropylene as it is reported to be nonpolar. Production of iso charge at temperatures $70^{\circ} \mathrm{C}$ to $110^{\circ} \mathrm{C}$, Table 3 is thus explained. The decrease of charge for samples prepared at above $90^{\circ} \mathrm{C}$ can be explained on the basis of increased conductivity at higher temperatures, because, as a result of higher temperatures the movement of ions becomes increasingly fast enough to escape trapping on or inside the crystallites at the time of cooling the sample while preparing the magnetoelectrets. Hence surface charge reduces as the temperature increases up to $110^{\circ} \mathrm{C}$.Because of heat energy; transfer of charges takes place at the metal-polymer boundary. These get trapped and frozen during cooling in the presence of magnetic field, giving rise to increase in idio charge density, which is predominant in iso charges density at higher temperatures.

In porous PP the charges can be injected to its volume through voids and trapped in particular sites or in free volume. As compared to the effect of magnetic field, the surface charge density is having more dependence on forming temperature because at elevated temperature there is possibility for more electrons to go into the traps due to large voids. This may increase the surface charge density. Porous materials became soft and show larger mechanical compatibility at elevated temperature during the polarizing process. At the same time, the coulomb forces of the mono charges situated at both sides of the pores with opening structure repel each other. This results in an expansion of the pores. When the sample is cooled to room temperature in presence of magnetic field, the expanded holes are solidified. It may be infer that, higher the polarizing temperature, larger is the diameter of the holes and higher the strength and stability of porous PP magnetoelectrets. The increase in surface charge density with magnetic field, Table 2 is due to charge trapped in the interior parts of the sample. The possibility of magnetic field creating traps in the substance has been observed by earlier researchers [8] when the field is increased, it helps to mobilise the charge in to deeper trap levels. This can be attributed to be the cause for the increase of surface charge with the increase of forming magnetic field. The charge in to deeper traps requires more energy for release from traps. Since the decay study is at room temperature, sufficient energy may not be available to the charges for release from the traps. Hence more charges are retained in the substance for quite a long period. Therefore it is basically the magnetic field, which sufficiently influences retentivity phenomenon. 
Table 2

Variation in iso-charge and idio-charge on the surface of magnetoelectret with respect to the forming parameters

\begin{tabular}{|c|c|c|c|c|c|c|}
\hline \multirow{3}{*}{$\begin{array}{l}\text { Forming } \\
\text { Magnetic } \\
\text { fields in } \\
\text { Tesla }\end{array}$} & \multicolumn{6}{|c|}{ Temperature } \\
\hline & \multicolumn{2}{|c|}{$70^{\circ} \mathrm{C}$} & \multicolumn{2}{|r|}{$80^{\circ} \mathrm{C}$} & \multicolumn{2}{|r|}{$100^{\circ} \mathrm{C}$} \\
\hline & $\begin{array}{l}\sigma_{\mathrm{iss}} \times 10^{-} \\
{ }^{10} \mathrm{C} / \mathrm{cm}^{2}\end{array}$ & $\begin{array}{l}\sigma_{\text {idio }} \mathrm{X} 10^{-} \\
{ }^{10} \mathrm{C} / \mathrm{cm}^{2}\end{array}$ & $\begin{array}{l}\sigma_{\text {isio }} \times 10^{-} \\
{ }^{10} \mathrm{C}^{-} \mathrm{cm}^{2}\end{array}$ & $\begin{array}{c}\sigma_{\text {idio }} \times 10^{-} \\
{ }^{10} \mathrm{C} / \mathrm{cm}^{2}\end{array}$ & $\begin{array}{l}\sigma_{\text {iso }} \times 10^{-} \\
{ }^{10} \mathrm{C} / \mathrm{cm}^{2}\end{array}$ & $\begin{array}{l}\sigma_{\text {idiox }} \times 10^{-} \\
{ }^{10} \mathrm{C}^{-} \mathrm{cm}^{2}\end{array}$ \\
\hline 0.75 & -0.584 & +0.259 & -0.584 & +0.259 & -0.551 & +0.162 \\
\hline 0.85 & -0.584 & +0.064 & -0.584 & -0.194 & -0.324 & +0.064 \\
\hline 0.95 & -0.551 & +0.032 & -0.811 & -0.227 & -1.558 & -0.649 \\
\hline 1.3 & -1.103 & +0.324 & -1.525 & +0.292 & -0.487 & +0.097 \\
\hline 1.5 & -0.746 & -0.162 & -0.649 & +0.129 & -0.551 & +0.162 \\
\hline
\end{tabular}

Table 3

Variation in initial charge, iso-charge and idio-charge on the surface of magnetoelectret with respect to the forming temperature

\begin{tabular}{|c|c|c|c|c|c|}
\hline \multirow{2}{*}{ Initial/Iso/Ido Charge } & \multicolumn{5}{|c|}{ Forming Temperature } \\
\hline & $70^{\circ} \mathrm{C}$ & $80^{\circ} \mathrm{C}$ & $90^{\circ} \mathrm{C}$ & $100^{\circ} \mathrm{C}$ & $110^{\circ} \mathrm{C}$ \\
\hline$\sigma_{\mathrm{N}} \times 10^{-10} \mathrm{C} / \mathrm{cm}^{2}$ & -0.779 & -1.233 & -0.389 & -0.389 & -0.454 \\
\hline$\sigma_{\mathrm{S}} \times 10^{-10} \mathrm{C} / \mathrm{cm}^{2}$ & -1.428 & -1.818 & -0.649 & -0.584 & -0.324 \\
\hline$\sigma_{\text {iso }} \times 10^{-10} \mathrm{C} / \mathrm{cm}^{2}$ & -1.103 & -1.525 & -0.519 & -0.487 & -0.389 \\
\hline$\sigma_{\text {idio }} \times 10^{-10} \mathrm{C} / \mathrm{cm}^{2}$ & +0.324 & +0.292 & +0.129 & +0.097 & -0.649 \\
\hline
\end{tabular}

\subsection{Surface Charge Decay Characteristics}

The quality of electret is mainly decided by its stability. The dynamics of the activation process, time and field dependence of the carrier concentration in the conduction band are responsible for the electret stability. The stability of electret charge is predicted by considering the rate of current carrier generation in activation processes and the rate of annihilation due to carrier capturing and recombination $[9,10]$. Some other factors that affect the stability of electrets are resistivity, degree of crystallinity, and the temperature at which the crystalline domains of the polymeric system used for electret fabrication; undergo transitional motions [11]. Mainly the charge decay occurs because of following reasons, 1) the deposition of compensation charge from the atmosphere, 2) the internal decay through conduction or drift, perhaps accelerated by invasive humidity. In order to find out the stability of electrets, surface charge decay of as prepared electrets was observed continuously for 30 days.

An exponential decay of surface charge is observed. Figure 1(a), (b). During formation of electret, surface and volume trapping of charge carriers is taking place. Impurities, broken polymer chains, or surface defect also the voids can act as surface traps and atomic sites in the molecular chain or crystalline domain boundary can act as the volume trap. The energy associated with these traps will be different and some act as deep trap and some as shallow trap. Therefore detrapping or decay of charges from these traps also may not be uniform and it may give rise to the exponential decay of surface charge as observed. The presence of humps on the initial stages of decay curve indicates that the decay of charge does not take place in a continuous manner. The sudden increase in the surface charge density may be because of the recapturing of the electrons in the shallow traps after they have been liberated due to thermal agitation at room temperature. The rapid decay on sample is attributed to compensation of the electret charges by atmospheric ions attracted by the electret. Internal charge decay processes in dielectrics are governed by conduction phenomena which depend on carrier mobility, carrier concentration etc [12]. 

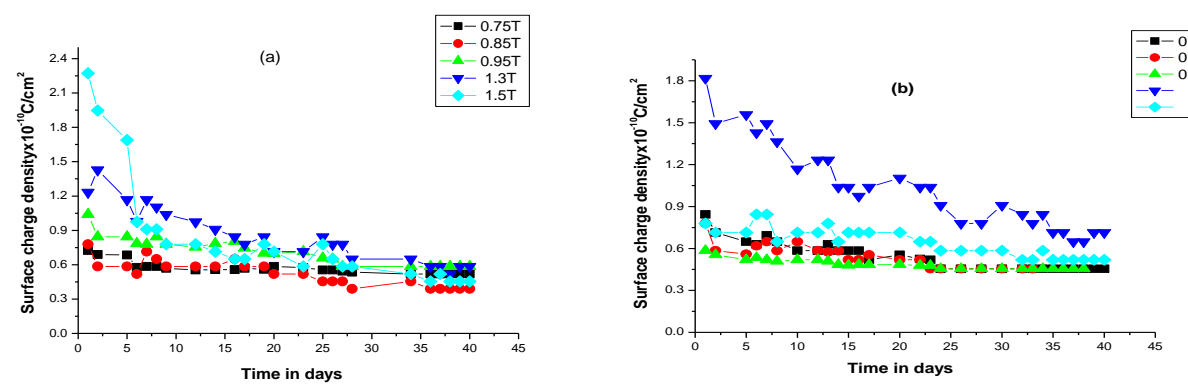

Figure 1 .Surface charge density decay of magnetoelectret prepared at $80^{\circ} \mathrm{C}$ at different forming fields. (a) Charge density decay from North surface of the magnetoelectret. (b) Charge density decay from South surface of the magnetoelectret

Table 4

Experimental decay time constant of magnetoelectrets prepared under different forming conditions. $\tau_{\mathrm{N}}=$ time constant for decay from North surface and $\tau_{\mathrm{S}}=$ time constant for decay from South surface.

\begin{tabular}{c|c|c|c|c|c|c}
\hline $\begin{array}{c}\text { Forming } \\
\begin{array}{c}\text { Magnetic } \\
\text { fields(T) }\end{array}\end{array}$ & \multicolumn{7}{|c}{ Temperature } \\
\hline & \multicolumn{2}{|c|}{$70^{\circ} \mathrm{C}$} & \multicolumn{2}{|c}{$80^{\circ} \mathrm{C}$} & \multicolumn{2}{c}{$100^{\circ} \mathrm{C}$} \\
\hline & $\tau_{\mathrm{N}}$ & $\tau_{\mathrm{S}}$ & $\tau_{\mathrm{N}}$ & $\tau_{\mathrm{S}}$ & $\tau_{\mathrm{N}}$ & $\tau_{\mathrm{S}}$ \\
\cline { 2 - 7 } 0.75 & 49 & 49 & 169 & 74 & 63 & 61 \\
0.85 & 84 & 86 & 46 & 84 & 52 & 117 \\
0.95 & 89 & 68 & 84 & 172 & 79 & 44 \\
1.3 & 34 & 37 & 35 & 40 & 79 & 116 \\
1.5 & 39 & 54 & 65 & 61 & 78 & 100 \\
\hline
\end{tabular}

By the method of successive reduction [13] of the semi log plot, the decay parameters can be calculated as follows.

$\sigma_{\mathrm{t}}=\sigma_{0} \cdot \mathrm{e}^{-\mathrm{t} / \tau}$

Where $\sigma_{0}$ and $\sigma_{\mathrm{t}}$ are the initial and instantaneous charge densities and $\tau$ is the decay time constant. On simplifying the equation we get,

$1 / \tau=\left(\ln \sigma_{0}-\ln \sigma_{t}\right) / t$

The variation in decay time constant of electrets prepared under different conditions was calculated and is given in Table 4, from the data it is observed that south surface decay time constant is higher which implies the better stability of surface charge compared to north surface. It is also observed that for the samples prepared at high temperature and high magnetic field the time constant for decay is high compared to that prepared at low temperature and magnetic field. This may be explained as there is more possibility for dipolar orientation at low forming temperature in comparison to the delocalization of excess charges. Therefore the surface charge of magneto electrets prepared at low forming temperatures shall originate more from the dipolar orientation which is contributing to idio-charge formation. Since idio-charge is having a faster decay rate compared to iso-charge, therefore samples prepared at low forming temperatures have faster decay rate than those prepared at high forming temperature.

\section{CONCLUSION}

Magnetoelectrets of porous polypropylene were prepared and dependence of surface charge density on temperature and magnetic field was observed by preparing samples at different forming conditions. A maximum surface charge density of $10^{-10} \mathrm{C} / \mathrm{cm}^{2}$ were observed in all the samples and there is no reversal in polarity of surface charge is obtained. Negative iso charge is obtained in all MEs. Due to combined action of heat and magnetic field, microscopic displacement of ions takes place giving rise to space charge polarization. It was found that the trend of surface charge decay at room temperature and variation in surface charge density with 
respect to the forming magnetic field and temperature are same in all MEs. The magnetoelectret state of porous polypropylene was studied and the material can be explored for further electret applications

\section{ACKNOWLEDGEMENTS}

One of the Authors Ms. Pragya Ojha is grateful to Director MANIT, Bhopal for providing scholarship.

\section{Reference}

[1] Heaviside C.K. Kwan, Dielectric Phenomena in Solids with Emphasis on Physical Concepts of Electronic Processes. Elsevier Academic Press, 2004.

[2] M. Eguchi, On the permanent electret. Phil. Mag. 49 (1925) 178-182.

[3] C.S. Bhatnagar, The magnetoelectret. Ind. J. Pure. Appl. Phys. 2 (1964)331-332

[4] G.M. Sessler, Electrets. Springer-Verlag, New York, 1980.

[5] M.L. Khare, C.S. Bhatnagar, Ind. J. Pure Appl. Phys. 8 (1970) 700-703.

[6] Gemant, APhil.Mag., 20,929-52(1935).

[7] HaswgawaH.\&Nakamura,M.,J.Phy.Soc.Japan,26,1362(1969).

[8] Ind.J Pure.Appl.Phys., 18,24,(1980)

[9] J.A. Ma1ecki, Linear decay of charge in electrets. Phys. Rev. B 59 (1999) 9954-9960.

[10] F. Galembeck, Carlos A.R. Costa, A. Galembeck, Maria do Carmo V.M. Silva, Supermolecular ionics:electric charge partition within polymers and other non-conducting solids. Ana. Acad. Bras. Cien. 73 (2001) 495-510

[11] A. Mishra, Studies of polymer electrets I. Factors governing the stabilities of homoelectrets obtained from poly(1-olefins). J. Appl. Poly. Sci. 154 (1982) 381-395

[12] G.M. Sessler, Electrets. Laplacian Press, Morgan Hill, CA, 1998.

[13] W. McMohans, Dielectric effects produced by solidifying certain organic compounds in electric or magnetic fields. J. Am. Chem. Soc. 78 (1956) 3290-3294 